\title{
Le manga est-il nécessairement lié à un contexte de production ? 1
}

\author{
Laurent Pendarias \\ ED 487- École doctorale de Philosophie, \\ Université Jean Moulin Lyon 3, \\ EA 4187-IrPhil

\section{Adrien Pendarias Chercheur indépendant}

Les dessins animés japonais, ou «japanimé », sont des produits de divertissement, pensés en fonction de la demande d'un public cible (tranche d'âge, genre du consommateur) et qui ont pu s'exporter aisément. L'exploitation par l'émission Club Dorothée (produite par AB Productions de 1987 à 1997), la réappropriation des codes graphiques pour des dessins animés français (Martin Mystère, Totally Spies) et enfin l'emploi de dessinateurs français par les studios japonais d'animation (Cédric Hérole) témoignent d'une réception favorable du public français pour des produits japonais, mais également de la capacité des Français à s'approprier un produit pour le revendre. Les commerciaux — producteurs, chargés d'édition, vendeurs et les créatifs - dessinateurs, scénaristes - peuvent reprendre à leur compte des innovations étrangères, malgré les différences culturelles, pour les adapter au marché.

La France est le deuxième pays consommateur de mangas, soit « le premier marché du manga à l'exportation. Mais bien qu'il s'en soit vendu, à son apogée en 2008, plus de 15 millions de volumes pour quelque 160 millions d'euros, les profits sont infimes pour les éditeurs nippons. Ces 160 millions représentent moins de $4 \%$ de leur chiffre d'affaires au Japon» (Bouissou 147). La France représente un marché plus intéressant que les pays asiatiques, ${ }^{2}$ qu'on pourrait supposer proches culturellement, ou que le Brésil,

\footnotetext{
${ }^{1}$ Les auteurs remercient les mangakas francophones qui ont accepté de participer à cette étude : Deruyck Vincent « Ancestral-Z », Guillaume Dorison «Izu » et « Aerinn ».

2 Les Coréens lisent des manhwa, des bande-dessinées coréennes dont le style se rapproche du manga sur certains points (noir et blanc, format, faible coût). Ce secteur représente $16 \%$ des ventes du marché de l'édition estimé à 4 milliards de dollars par la KPA (Korean Publisher Association). On pourrait donc supposer que le public coréen est plus à même que le français d'acheter des mangas japonais, toutefois les faibles ventes de ces derniers peuvent s'expliquer par des causes historiques. À noter l'existence de lois en Corée du Sud interdisant l'import de produits culturels japonais entre 1945 et 1998. Si, en droit, ces limitations sont levées, de fait elles provoquent encore des incidents : en 2014, la chaîne publique coréenne KBS refuse la chanson coréenne «Uh-ee » du groupe de pop coréenne Crayon Pop au motif qu'elle contient un mot japonais. Au-delà des stratégies économiques protectionnistes le contexte historique spécifique explique ces heurts : dans la première
} 
qui accueille la deuxième communauté nippone, et concentre quatre fois plus de ventes que les USA, partenaires économiques historiques du Japon depuis 1945. On peut donc avancer que la France offre le terreau le plus favorable au manga.

Le Japon organise la production de ces «bande dessinées » dans des magazines de prépublication (Weekly Shônen Jump, V Jump, Jump Square, Margaret, etc.), vendus à bas prix (240 yens environ). Les séries, diffusées sous la forme d'épisodes, hebdomadaires ou mensuels, perdurent si elles obtiennent du succès auprès de leur public et sont par la suite republiées sous forme de tankôbon, des petits formats de meilleure qualité pour les collectionneurs, équivalents aux albums français.

La culture manga a pu influencer l'Occident dans les années 80 via les animés (Goldorak, Albator, Dragon Ball) et Glénat a fait traduire et éditer Akira en français en 1990. Dès lors, on pourrait supposer qu'une génération de créateurs français a grandi en contact avec les nouveaux codes graphiques.

En 2006, débute en France Shogunmag, un magazine de prépublication mensuel calqué sur le modèle de diffusion japonais et publié par les Humanoïdes Associés dans un contexte favorable. Ce dernier se décline en trois magazines, respectivement Shogun Shōnen, Shogun Seinen et Shogun Life, reproduisant ainsi la tactique commerciale japonaise consistant à viser des niches précises en fonction de l'âge et du sexe. Compte tenu de la situation de monopole commercial, ces magazines auraient dû remporter un succès, mais ont disparu peu à peu, quittant le papier pour le numérique, puis espaçant les publications.

L'arrêt de Shogunmag malgré un contexte économique favorable et une génération de créatifs motivés amène à s'interroger sur les particularités du format « manga ».

moitié du vingtième siècle le Japon impérialiste avait annexé la Corée, la Chine du Nord-Est et une partie de la Russie. 


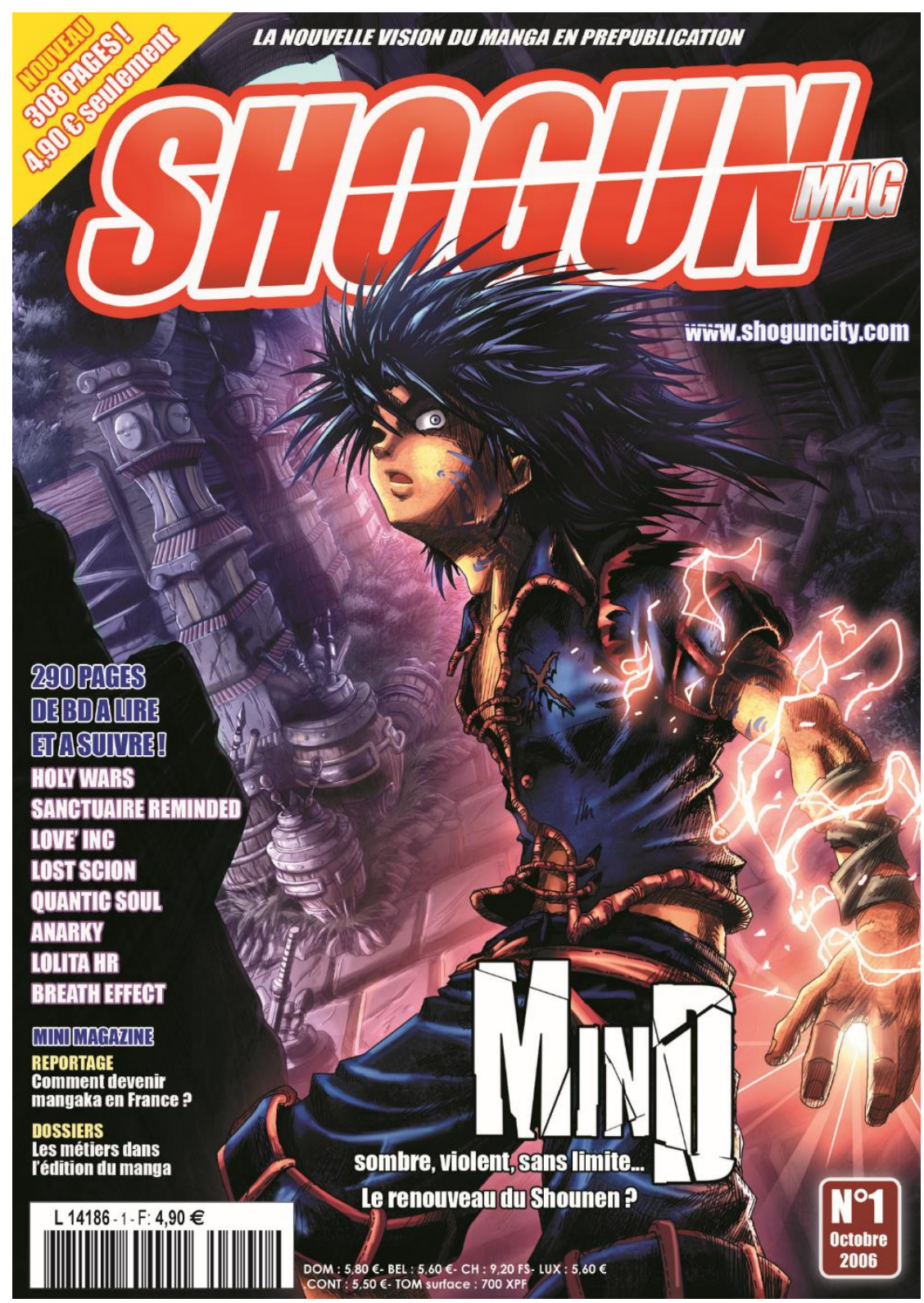

Shogunmag, volume 1 - Collectif

(C)2016 Humanoids, Inc. Los Angeles.

Si la France conçoit le développement d'œuvres pour leur seule existence, ${ }^{3} l^{\prime}$ histoire du manga au Japon résulte davantage d'une série de défis

3 Le philosophe Kant définit comme critère de l'œuvre d'art dans Critique de la faculté de juger (1790) sa gratuité au sens de «non-utilité » par opposition à l'objet créé par l'artisan. Le mouvement de l'art pour l'art apparait au dix-neuvième siècle et défend la thèse que l'existence de l'œuvre d'art se justifie par elle-même. Elle n'est pas un moyen au service de la politique, de la morale ou du commerce. Cf. Théophile Gautier, préface de Mademoiselle de Maupin (1834). Enfin, au vingtième siècle la philosophe Hannah Arendt réactualise cette 
face aux contraintes techniques et économiques poussées d'où une recherche d'efficacité assumée à la fois dans les autobiographies des auteurs, mais aussi les communications d'entreprise. Les Japonais eux-mêmes disent du manga qu'il «n'est pas un art » (Bouissou 191) alors qu'ils reconnaissent ce statut de bijutsu (d'art) à la bande dessinée franco-belge.

Le manga est conçu pour un public cible avec un objectif de rentabilité assumé. ${ }^{4}$ La création des ateliers à partir des années soixante permet aux auteurs de travailler et donc de produire plus rapidement. L'apparition des chargés d'édition - responsables de l'encadrement des auteurs - sert également à fiabiliser la réalisation. Enfin, la création des systèmes de votes, de consultation des lecteurs, permet à ces derniers d'avantager leur série favorite, facilitant ainsi la longévité des séries les plus appréciées et donc les plus rentables. S'il fallait caricaturer ce dernier point, pour marquer la différence avec la vision européenne, ce serait d'affirmer que le meilleur chanteur est celui qui obtient le plus de votes à Star Academy ou qui vend le plus d'albums au lieu de juger la qualité esthétique des contenus produits, or la vision européenne privilégie un Proust à un George Ohnet (auteur de romans populaire de son vivant, tombé dans l'oubli).

Peut-on faire du manga comme on fait de la bande dessinée ? Aux yeux d'un Européen, habitué aux codes de la bande dessinée franco-belge, considérée comme un objet artistique, ${ }^{5}$ le manga semble être une autre forme de bd qui différerait seulement par ses codes graphiques et ses références culturelles. Cependant l'arrêt de Shogunmag et le petit nombre de manfra manga français - à succès, conduit à considérer la thèse opposée selon laquelle le manga ne serait pas une autre forme d'art facilement reproductible, mais un système industriel et commercial rationnellement organisé.

conception de l'art gratuit dans La Crise de la Culture (1961-1968) en défendant la thèse que l'œuvre s'oppose à l'objet d'usage, au produit de consommation. À noter enfin la théorie du réalisateur-auteur dans le cinéma qui fait du réalisateur l' "auteur » de son film, tout comme l'architecture reconnait ce statut à l'architecte et non au promoteur, alors qu'il s'agit d'une œuvre collective. En France, ce courant est formalisé par François Truffaut, en 1955 dans les Cabiers du Cinéma, qui initie « la politique des auteurs».

${ }^{4}$ Dans son $œ u v r e$ autobiographique Gekiga Fanatics, Masahiko Matsumoto présente le personnage de l'éditeur Hinomaru comme un personnage qui ne cesse de répéter qu'il faut «vendre » (25 occurrences). Malgré le caractère romancé, ce témoignage d'un pionnier insiste volontairement sur l'impératif économique. Enfin l'objectif des héros mangakas de la série Bakuman (voir ci-dessous) consiste à devenir les numéros 1 en termes de votes et de ventes.

5 Bien que la bande dessinée soit parfois considérée en France comme un objet de divertissement destiné aux enfants, l'existence de musées dédiés et de prix témoignent d'une certaine reconnaissance. Enfin, en France, les auteurs de bande dessinée travaillent avec le statut d' "artiste auteur » et sont enregistrés sous cette appellation auprès des organismes auxquels ils cotisent comme l'URSSAF ou l'AGESSA. La catégorie socio-professionnelle des auteurs de $\mathrm{BD}$ est rattachée à celle des artistes et non des artisans ou des employés. 
Ce paradoxe nous conduit à poser la problématique suivante : Le manga est-il nécessairement lié à son contexte de production?

Plusieurs hypothèses ont déjà été avancées dans le cadre d'analyses antérieures pour tâcher de rendre compte de l'impossibilité des artistes français à copier les mangas. Sans prétendre à l'exhaustivité, nous résumons ci-dessous les principales :

- L'incompréhension des codes graphiques : une explication possible serait la non-appropriation des codes graphiques par la génération française. Malgré l'arrivée des animés japonais dès la fin des années 70, le premier manga traduit et édité, avec succès, par Glénat est Akira en 1990. Les dessinateurs français ont-ils eu le temps d'intégrer ces nouveaux codes si différents de la bande dessinée franco-belge ? Alors que les Européens ont majoritairement appris à dessiner avec le " gaufrier», la planche organisée en cases de même taille, peuvent-ils s'approprier un style qui ne respecte pas les cases voire s'en affranchissent? Créer des mangas exige de connaittre les codes propres du support et leur assimilation.

- Les barrières morales : que penser du héros de Kozure ôkami (Lone wolf and cub), ${ }^{6}$ de sa quête de vengeance et de ses victimes innocentes? Le personnage cherche le meifumado, la voie de l'enfer. Quel exemple présente le héros de Shamo (Coq de combat) ? Après avoir tué ses parents, il entre dans le monde des arts martiaux et cherche la victoire à tout prix, y compris par la triche et le dopage. Ces héros «à voie» ne correspondent pas à la morale judéochrétienne. Au-delà du problème de la violence représentée, déjà dénoncée par Ségolène Royal dès 1989 puis par Pascal Lardellier dans Le Monde diplomatique, c'est la non-compatibilité des valeurs morales qui empêcherait les Français de composer des histoires comme les Japonais. S'il existe des héros de BD franco-belges qui ne sont pas des modèles de vertu, il demeure difficile de comprendre les concepts moraux asiatiques avec une interprétation européenne. S'ajoute à cela le passé de ce pays associé à l'Allemagne nazie, ennemi historique de la France, qui pourrait expliquer sa perception négative par une partie de la population. Ainsi Ozamu Tezuka reconnât dans

${ }^{6}$ Lone Wolf and Cub, littéralement « le loup accompagné de son petit » est un gekiga manga historique écrit par Kazuo Koike et dessiné par Goseki Kojima. Prépublié entre 1970 et 1976 dans le magazine Weekly Manga Action de l'éditeur Futabasha, il raconte la quête de vengeance d'Ogami Ittō, l'exécuteur déchu du shogun.

7 Coq de combat est un manga écrit par Izō Hashimoto (tomes 1 à 25), et dessiné par Akio Tanaka (puis écrit également depuis le tome 25). Il a été prépublié entre 1998 et 2003 dans le magazine Weekly Manga Action de l'éditeur Futabasha, avant d'être prépublié entre 2005 et 2015 dans le magazine Evening de l'éditeur Kōdansha. 
son autobiographie son admiration passagère pour les jeunesses hitlériennes :

Pendant la guerre, je me souviens que pour nous, les Japonais, l'idée de l'Allemagne nazie était très positive. Nous étions surtout admiratifs des Jeunesses hitlériennes, les "Hitlerjugend", que nous considérions comme un groupe de jeunes qui vouaient leur vie au Führer. Oui, pendant une certaine période, j'ai envié les enfants allemands de pouvoir consacrer leur vie à quelque chose qui me paraissait aussi beau. (96).

- Après sa défaite et l'annonce d'un armistice, la France a traversé une période dite de « collaboration » avec l'Allemagne nazie, via le régime de Vichy de 1940 à 1944, contestée par le mouvement de la «Résistance », symboliquement daté de l'appel du 18 juin 1940 du Général de Gaulle tandis que le Japon a choisi de se positionner comme allié officiel de l'Allemagne nazie depuis la signature du Pacte tripartite le 27 septembre 1940 jusqu'à la défaite de 1945.

- La censure française : la Loi du 16 juillet 1949 sur les publications destinées à la jeunesse « de la protection de l'enfance » censure tandis que les auteurs japonais abordent tous les sujets y compris la violence et la mort. Le droit aurait donné plus de libertés aux Japonais. Cette hypothèse "juridique » fait l'impasse sur des problèmes similaires rencontrés au Japon : outre la rafle des revues ero-guro organisée en 1955, par la préfecture de police, rapportée par le mangaka Masahiko Matsumoto, Ozamu Tekuka déclare aussi dans son autobiographie essuyer des attaques :

Les critiques les plus virulentes portaient sur l'idée que cette histoire était absurde : «C'est totalement idiot! Un robot, ça n'existe même pas! »[...] On me montrait du doigt comme si j'étais un rêveur imbécile qui mettait des absurdités dans la tête des enfants. Juste à ce moment-là, à Hokkaidô je crois, un enfant a sauté de la fenêtre du premier étage déguisé en Astro et s'est gravement blessé. Les journaux se sont emparés de l'affaire, et les intellectuels patentés se sont mis à crier haro sur les mangas. (144).

- Le goût de l'authenticité : une explication possible de cette nonimplantation serait que les Français veulent de l'exotisme, donc des histoires japonaises, le produit original, tout comme les consommateurs désirent du "champagne » français authentique aux quatre coins du globe, plutôt qu'une contrefaçon. Les consommateurs français ne souhaiteraient pas acheter des manfras mais des mangas japonais, et par conséquent le manga serait inséparable de son contexte d'origine. Les créateurs français se rejoignent sur ce problème: pour le dessinateur Ancestral Z «souvent les lecteurs de mangas français sont très attachés au côté exotique et au style atypique des mangas. (le fait que cela se passe au Japon par exemple...)». («Entretien du 27 janvier $2016 »)$. Le 
scénariste Guillaume Dorison, raconte qu'il a souvent vu au cours des salons des lecteurs intéressés par les manfras, accrochés par le style graphique des couvertures et des pages, mais qui reposent dès qu'ils découvrent que c'est «français» («Entretien du 8 février $2016 »)$. Enfin la mangaka Aerinn conclut

[...] habitués à la rapidité japonaise, à la qualité des émotions ressenties... les lecteurs français sont restés jusqu’à présent très fermés aux créations françaises, qu'ils perçoivent comme de pâles copies de leurs séries préférées [...] Mais faute d'avoir maitrisé parfaitement les codes du manga et les véritables objectifs des éditeurs japonais, les essais qui ont été faits sur les manfras ont pour la plupart été des échecs, [...] donnant ainsi raison au public français qui a alors associé la création française à un niveau médiocre. («Entretien du 1er février $2016 »)$.

- L'absence d'audace, le problème du «lissage des scénarios » : alors que les Japonais développent des histoires pour toucher un public très ciblé (les adolescentes par exemple), « en France, la recette pour fabriquer des best-sellers découle mécaniquement de la petite taille du marché. Pour réaliser de très grosses ventes, les éditeurs doivent privilégier une stratégie attrape-tout, afin, comme feu l'hebdomadaire Tintin, de séduire les lecteurs de "7 à 77 ans" " (Bouissou 199). Alors que les mangas japonais s'inscrivent dans des genres identifiables pour cibler des niches (Shonen pour adolescents, Shojo pour adolescentes, etc.), voire des sous-genres inconnus en France, ${ }^{8}$ les scénaristes français choisiraient des histoires censées plaire à tous et qui au final plairaient de moins en moins.

- L'incongruité du techno-animisme en France. Les Japonais attribuent des esprits à tous les étants y compris les récents objets technologiques. Pour Trécourt, le monde occidental désenchanté, marqué par un rationalisme cartésien, et un monde japonais constamment imprégné de magie malgré son évolution technologique peuvent difficilement se retrouver.

\footnotetext{
${ }^{8}$ Kodomo (public jeune, moins de dix ans), Seinen (jeunes hommes adultes), Josei (pour femmes actives), Yaoi (histoire homosexuelle entre hommes destinée aux lectrices) à ne pas confondre avec le courant Gay (histoire homosexuelle entre hommes destinée aux lecteurs), Yuri (histoires homosexuelles féminines pour lectrices), Ero-guro (érotisme macabre et/ou grotesque), etc., jusqu'à des catégories interdites en France depuis le 5 août 2013 comme le Lolicon (productions érotiques mettant en scène des mineures).
} 


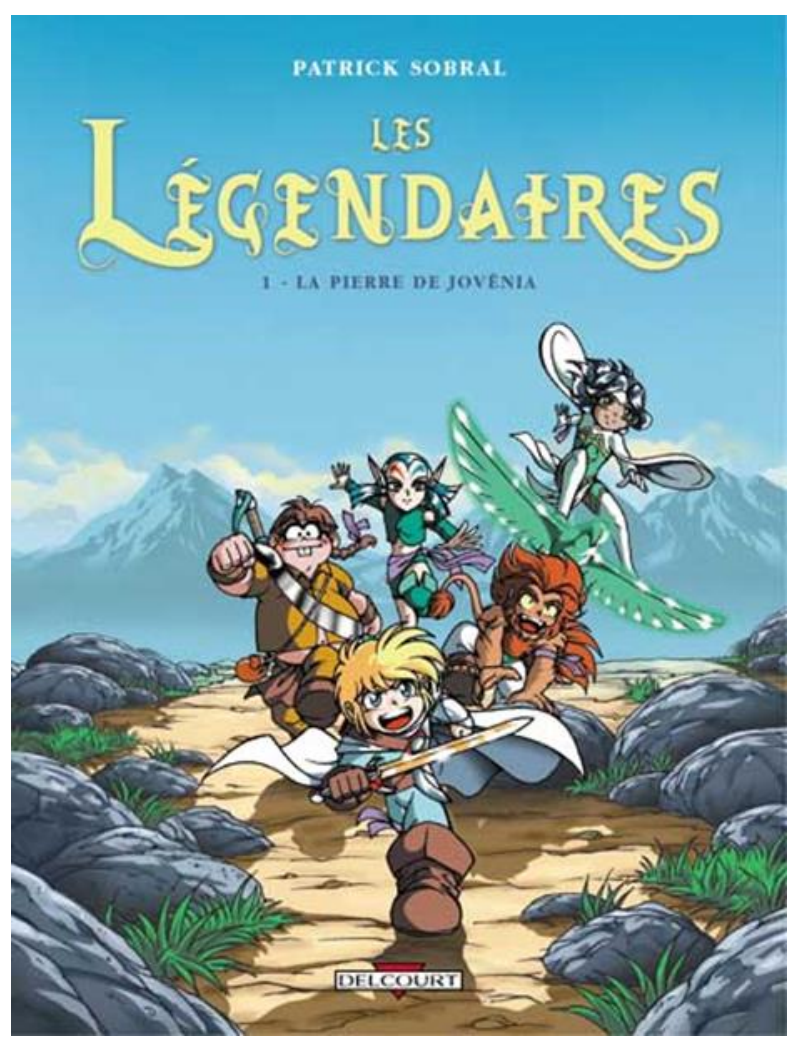

Les Légendaires, volume 1 - Patrick Sobral

(C) Éditions Delcourt, 2004

- Enfin, si on interroge les créateurs, ils soulignent aussi le rôle des éditeurs français dans ce développement ralenti du manfra : Dorison explique que "dans les années 90 les éditeurs ne voulaient pas publier de mangas. Quand quelqu'un venait leur proposer un projet, ils l'envoyaient faire de la BD » (Entretien du 8 février 2016) ainsi Delcourt a publié dans les années 2000 les séries Les Légendaires de Patrick Sobral et La Rose écarlate de Patricia Lyfoung, au format franco-belge, mais à l'esthétique manga. Aerinn renchérit sur les différences entre les deux pays : au Japon l'éditeur accompagne, corrige et guide l'apprenti mangaka tandis qu'en France l'artiste, isolé, est sommé de produire immédiatement une œuvre comparable aux classiques japonais (Entretien du 1er février 2016)

$\mathrm{Si}$ le format «manga» s'avère indissociable de son contexte de production, alors il ne peut s'exporter et se réimplanter en France à la façon d'une plante radicante qui se déplacerait en plantant ses racines dans son nouveau milieu. Notre thèse ne se résume pas à constater un état de fait, à savoir un échec partiel, mais à comprendre quelles caractéristiques du manga, chez les créateurs, les éditeurs ou le public, ont été occultées ou incomprises, notamment la dimension et l'organisation rationnellement agencée du manga 
au Japon contre son approche occidentale, renvoyant dos à dos la conception "méfiante», et réductrice des années 80 symbolisée par Ségolène Royal, et l'optimiste de "la nouvelle manga », cherchant une dimension artistique dans la manga, incarnée par Boilet.

Cette thèse se développera en suivant quatre étapes : d'abord l'analyse du manga Bakuman, comme journal interne de la compagnie Shüeisha, d'après le cadre d'analyse du knowledge management japonais d'Ikujiro Nonaka, permettra d'expliciter l'organisation industrielle du manga au vingt-et unième siècle. Puis l'examen du déterminisme historique permettra d'éclairer comment l'histoire du Japon a conditionné celle du manga. Ensuite, l'étude des expédients dans le dénuement montrera comment les Japonais ont su pallier le manque de moyens pour construire une activité rentable. Enfin, la conclusion portera sur l'analyse empirique des échecs, mais aussi des succès français.

En prenant le parti d'envisager le manga sous l'angle industriel, il convient de se tourner d'abord vers les analyses managériales, avant d'envisager toute question esthétique: Tarun Khanna (70-80) défend la thèse dans Harvard Business Review que les meilleures pratiques de management ne peuvent pas s'exporter dans tous les pays. On ne peut appliquer de manière uniforme un système théorique qui a fonctionné dans un pays sans prendre en compte la spécificité de ce nouveau pays. Cette analyse expliquerait pourquoi la copie du manga par les Français n'a pas fonctionné.

Dans la lignée des travaux d'Anthony Mayo et Nithin Nohria, ${ }^{9}$ Tarun Khanna suppose que le transfert d'un marché d'une zone culturelle à une autre nécessite une « intelligence contextuelle : la capacité à comprendre les limites de notre connaissance et à adapter cette connaissance à un environnement différent de celui dans lequel elle a été développée » (72).

Les Européens ont pu réduire le manga à une forme de bande dessinée médiocre et « méprisable », copie de moindre qualité en raison de 1) son prix inférieur, 2) la médiocrité graphique (noir et blanc sur papier jetable) et 3) son contenu (violence, absurde, fantastique). Les Franco-belges se croiraient alors capables de copier voire de sublimer le manga, mais échouent parce que le manga n'est pas qu'une bande dessinée en noir et blanc qui se joue des cases, mais toute une industrie organisée rationnellement qui a su produire des œuvres reconnues et primées. Ainsi le festival d'Angoulême a

9 Lire notamment sur le sujet Mayo Anthony, Nohria Nitin et Rennella Mark. 2009; Mayo, Anthony, Nohria, Nitin et Singleton L. G. 2007 ; Mayo, Anthony et Nohria, Nitin. 2005. 
récompensé plusieurs auteurs japonais ${ }^{10}$ et des musées réputés (Guimet ${ }^{11}$, Pompidou $^{12}$ ) ont organisé des expositions autour du manga.

\section{Bakuman, le journal interne de Shüeisha}

Bakuman est un manga paru dans l'hebdomadaire japonais Weekly Shonen Jump entre 2008 et 2012, publié en France sous la forme de vingt volumes reliés ${ }^{13}$ et adapté en dessin animé puis en film. Comme tout shonen, il respecte un cahier des charges prévisible : les héros sont deux adolescents, endurants, prêts à s'entraîner et à se battre pour devenir les numéros un du Japon. Ici, l'originalité tient au but : devenir mangakas, c'est-à-dire auteurs de mangas.

\section{a) Encore un manga sur les mangas?}

Ce n'est pas la première fois qu'un manga met en scène un ou des mangakas : Akira Toriyama a publié en 1984 la série Hetappi. Crummy Manga Lab (L'Apprenti mangaka), en quatre épisodes pour donner les conseils essentiels aux lecteurs pour la réalisation d'un manga.

Bakuman perpétue cette tradition en allant néanmoins plus loin que ses prédécesseurs, car il explique, dans les moindres détails, tous les aspects concrets du métier et notamment les salaires, les délais et les formats. ${ }^{14} \mathrm{Au}$ lieu de se concentrer sur un personnage, le manga fait la part belle à l'organisation et explique le rôle de chaque participant, du simple assistant chargé de dessiner les décors jusqu'au directeur de publication du Jump en passant par les éditeurs, dont le rôle est peu connu du grand public.

\section{b) Un manga informatif?}

\footnotetext{
10 Grand prix de la ville d'Angoulême 2015 : Katsuhiro Ōtomo. Grand prix de la ville d'Angoulême 2013 Akira Toriyama. 2001 : Prix du jury œecuménique de la bande dessinée décerné à Jiro Taniguchi pour Le Journal de mon père. 2003 Alph-Art du meilleur scénario au Festival d'Angoulême 2003 pour le tome 1 de Quartier lointain. 2005 Prix du dessin du Festival d'Angoulême 2005 pour le tome 2 du Sommet des dieux. Chevalier de l'Ordre des Arts et des Lettres. Décoration remise par Frédéric Mitterrand à Tokyo le 15 juillet 2011.

11 «Samouraïs, moines et ninja. Quand le manga revisite l'histoire japonaise. » $1^{\text {er }}$ juillet - 9 août 2010.

12 «Salon manga » 8 février - 28 mai 2012.

${ }^{13}$ Écrit par Tsugumi Ohba et illustré par Takeshi Obata, le manga a été publié en France aux éditions Kana.

${ }^{14}$ Le manga indique explicitement à travers les dialogues et les notes de bas de page des informations concrètes : la planche est payée 12000 yens (soit environ 110 euros), l'artiste doit systématiquement fournir le chapitre suivant deux semaines à l'avance, les premiers chapitres et histoires complètes comptent une cinquantaine de planches contre dix-neuf pour les chapitres hebdomadaires.
} 
La société japonaise est parfois qualifiée de "Société de l'information » (Rieu 185-216) jôhồ shakai, et en 1986, le quotidien économique Nibon Kenæai Shinbun invente le "manga informatif» (jôhô manga) (Bouissou 123) avec Nibon manga keizai nyûmon (Les secrets de l'économie japonaise en bande dessinée $)^{15}$, qui se décline ensuite en salaryman manga et business manga. L'idée est de mettre en scène des employés d'entreprise afin de communiquer des informations pratiques via le détour de la narration. Par exemple, le héros Shima Kôsaku des mangas éponymes (le titre de la série change selon le grade de l'employé) travaille pour Hatsushiba Electric (représentation fictive de Panasonic). De la même façon Shüeisha publie Bakuman, l'histoire des employés de Shüeisha dont l'objectif assumé est de devenir numéro un des ventes.

Sachant que les auteurs ont réalisé Bakuman après le best-seller Death Note, ${ }^{16}$ ce serait opérer une lecture incomplète que de le réduire à son contenu, un outil documentaire, en manquant ainsi une dimension essentielle : la vision nippone du management «à la japonaise ». Nous posons l'hypothèse que les auteurs du manga ont voulu illustrer la théorie d'Ikujiro Nonaka et d'Hirotaka Takeuchi concernant la création et la diffusion du savoir en entreprise.

\section{c) Le management à la japonaise selon Nonaka et Takeuchi}

Ikujiro Nonaka et Hirotaka Takeuchi écrivent et publient en 1995 leur pièce maitresse : The Knowledge-Creating Company (La Compagnie créatrice de savoir), ${ }^{17}$ marquant une étape essentielle dans la naissance du management de la connaissance.

Le livre s'adresse au lecteur occidental et traite d'une problématique qui interpelle les Américains: pourquoi les sociétés japonaises sont-elles si compétitives? Pour Nonaka et Takeuchi, leur avantage réside dans la création de nouvelles connaissances, permise par un certain système de management. Alors que les Occidentaux tendent à concevoir ce dernier sur un modèle pyramidal (avec un chef au sommet qui distribue des ordres aux échelons inférieurs), les Japonais présentent le management de connaissances comme

15 マンガ日本経済入門 (Les Secrets de l'Economie Japonaise) est un manga créé en 1986 par Shotaro Ishinomori et raconte de manière romancée les aventures de deux managers aux visions divergentes.

${ }^{16}$ Death Note est un manga shonen, scénarisé par Takeshi Obata et dessiné par Tsugumi Ôba, prépublié dans le magazine Shônen Jump de 2004 à 2006. La version française diffusée par Kana comprend 13 volumes. Avec plus de trente millions d'exemplaires vendus, le manga a donné naissance à une série animée de 37 épisodes, 3 films live, une série live, une pièce de théâtre, un roman et trois jeux vidéo.

17 Traduction et édition française dirigée par Marc Ingham (1997). 
l'interaction organisée de savoirs tacites et explicites, reprenant la distinction du philosophe américain Michael Polanyi ${ }^{18}$.

\section{d) Distinguer savoir tacite et savoir explicite}

On peut poser des mots sur les savoirs explicites, ou les écrire pour les conserver et les transmettre. Par exemple, nous savons que «François Hollande est devenu Président de la République française en $2012 »$ ou que « $2+2=4$ ». Pour Nonaka, il s'agit du mode dominant de la tradition philosophique occidentale. En revanche, les Japonais accordent plus d'importance au savoir tacite (a tacit knowledge) qui « ne peut être exprimé par le langage formel, car il s'agit d'un savoir personnel “'incorporé »" dans l'expérience individuelle et qui inclut des facteurs invisibles comme les croyances personnelles, la perspective et le système de valeur $»^{19}$. Par exemple, je sais « cuire une omelette », je maîtrise l'action de la cuisson, mais je serais incapable d'expliquer avec un discours toutes les actions concomitantes qui me permettent de cuire de manière homogène le plat.

Pour un Occidental les mots «data», «information» et "knowledge » semblent interchangeables. Les compagnies japonaises ont une approche différente : l'explicite n'est que le sommet de l'iceberg du savoir, car le tacite, par définition personnel et non formulable, ne peut se communiquer ou se partager. Pour reprendre les exemples précédents, on peut facilement transmettre à une tierce personne une information explicite comme une formule mathématique, « $2+2=4$ », toutefois il ne suffit pas de dire à quelqu'un comment «cuire une omelette » pour qu'il acquière ce savoir, parce que le tacite est personnel, propre à la personne qui le pratique.

Nonaka et Takeuchi présentent un modèle intitulé «la spirale du savoir » qui montre comment rendre le savoir tacite explicite, et inversement, en passant par quatre modes de conversion (62).

- L'externalisation : Certains personnages possèdent les savoir-faire, mais les phases de discussion (entre auteurs ou entre auteurs et éditeurs) permettent d'expliciter ce qui peut paraître évident pour les pratiquants, mais pas pour les observateurs. Par exemple, dans le volume 3 le personnage d'Eiji Nizuma, présenté comme le numéro

\footnotetext{
18 Michael Polanyi (1891-1976) fut un chimiste et philosophe américain. Il se propose d'expliquer le fonctionnement de la recherche scientifique par les savoir-faire des chercheurs et développe ainsi une distinction entre savoirs explicites "explicit knowledge » et savoirs tacites « tacit knowledge ».

${ }^{19}$ Ils précisent dès la préface la définition de cette notion clé qu’est le savoir tacite : « [...] which is hard to articulate with formal language. It is personal knowledge embedded in individual experience and involves intangible factors such as personal belief, perspective, and the value system ». (viii) (Traduction personnelle).
} 
un et le rival à dépasser, comprend l'importance des nemus, ${ }^{20}$ à savoir les brouillons préparatoires, après échanges verbaux.

- La combinaison de connaissances explicites s'opère dans les dialogues; par exemple, au chapitre 28, l'éditeur fournit au scénariste des romans policiers afin de fournir des idées. Un troisième personnage lit ces livres et rédige des résumés : les informations explicitées peuvent se figer dans des écrits et les personnages échangent ces dernières pour penser la conception du manga policier Trap.

- L'internalisation (ou incorporation), l'acquisition du savoir implicite ne peut s'opérer uniquement par le dialogue et doit advenir par l'apprentissage (le savoir tacite s'acquiert par l'expérience). Ainsi le scénariste Takagi consacre une part importante de son temps à échanger avec l'éditeur, à manipuler des idées explicites. A contrario, le personnage de Mashiro, le dessinateur, s'impose des plages de travail consacrées à la seule maitrise de la technique, tout comme un pianiste se doit de répéter ses gammes pour conserver son niveau.

- La socialisation s'observe enfin dans les équipes. Pour Nonaka et Takeuchi, une façon d'acquérir le savoir tacite est de travailler aux côtés de quelqu'un qui le maîtrise et ainsi on finit, à force d'observation, par le copier. Le manga insiste sur le rôle des équipes : le mangaka est épaulé par deux à cinq assistants qui réalisent une partie du travail technique. Ces derniers acquièrent une expérience et une compétence non seulement par leur pratique, mais aussi par imitation puisqu'ils déclarent avoir progressé «au contact ${ }^{21}$ des professionnels expérimentés.

${ }^{20}$ Les nemus sont des brouillons préparatoires crayonnés qui permettent de fixer les cadres et le placement des personnages des futures planches qui seront encrées. Leur court temps de réalisation permet de prototyper un chapitre et de discuter des améliorations/corrections possibles avant de passer à la réalisation définitive.

${ }^{21}$ Le manga Bakuman insiste à plusieurs reprises sur ce point. Le chapitre 22 reprend cette distinction entre savoir explicite exprimé à l'oral et apprentissage du tacite par imitation quand le héros Mashiro échange avec Fukuda sur leur rival, Eiji, le jeune prodige :

- Contrairement à un mangaka qui aurait de l'expérience, Nizuma n'est absolument pas disposé à enseigner quoi que ce soit.

- Je ne demande rien. Je veux juste le regarder.

Le même débat se reproduit entre Eiji (porteur d'un savoir tacite invisible) et Mashiro (en cours d'apprentissage) :

- Je suis venu ici en espérant pouvoir apprendre quelque chose.

- Quoi ? Il y aurait quelque chose à apprendre de moi ?

- Peut-être, oui... Si tu me laisses te regarder dessiner.

$\mathrm{Au}$ chapitre 24 le héros conclut que ses apprentissages viennent du personnage Nakaï (33 ans), l'assistant le plus vieux et le plus expérimenté : 
La thèse de Nonaka et Takeuchi est originale à plus d'un titre :

- Ils défendent l'idée que la création de savoir dans l'entreprise ne dépend pas uniquement des chefs ou des hommes de terrain, mais de tous les individus, à tous les niveaux (dirigeants, managers intermédiaires et techniciens).

- De même, les dirigeants doivent donner une vision, mais laisser de l'autonomie aux équipes de recherche.

- L'entreprise, par son organisation, peut créer du savoir, mais la source de création du savoir est et sera toujours l'humain.

- Ce savoir prend naissance dans l'humain, souvent sous forme tacite. Il est donc nécessaire de passer par les quatre phases de conversion pour transformer et faire évoluer ce savoir. Par exemple, l'entreprise Canon utilisait un journal interne pour faire circuler les connaissances au sein de l'entreprise. De même, la compagnie Matsushita publia le magazine "Hot-Line», pour diffuser le savoir explicite aux travailleurs des différents départements. Or, la Shüeisha dans laquelle évoluent les héros de Bakuman met en scène, via le manga qu'elle publie, les pratiques de l'entreprise et ses évolutions.

\section{e) Diffuser les idées par le biais de la narration}

Nonaka insiste sur la difficulté à transmettre des connaissances tacites puisque, par définition, on ne peut pas les exprimer à l'aide de phrases ou de formules. Il faut donc recourir aux analogies, aux métaphores ou encore aux histoires. Bakuman parvient, par le biais de la narration, à transmettre des messages essentiels sur les techniques et sur l'organisation du travail au sein du magazine Weekly Shonen Jump (détenu par la compagnie Shüeisha).

À la question de savoir qui crée le savoir en entreprise, le manga prend le parti de montrer l'implication de chaque échelon : assistant dessinateur, auteur, éditeur, capitaine d'équipe et directeur de publication..$^{22}$ Le rôle de chaque membre de l'équipe est souligné et mis de l'avant pour dépasser la vision mythique et fantasmée de l'artiste-créateur ${ }^{23}$.

Bakuman montre comment les dirigeants peuvent impulser, donner une vision d'ensemble, tout en laissant de l'autonomie aux équipes créatives : les

- Je reconnais qu'ici, c'est en regardant travailler M. Nakai que j'ai appris le plus de choses.

22 Le manga fait également apparaître, au chapitre 37, M. Torishima, le véritable directeur général, mêlant ainsi des personnages existants à la fiction.

23 Pour l'Occident, Nietzsche avait déjà critiqué dans Humain, trop bumain ce mythe romantique du génie, répandu dans l'Europe du XIX ${ }^{\mathrm{e}}$ siècle, et de la personnalité extraordinaire de l'artiste. 
éditeurs et directeurs ne cessent d'encourager et de guider les artistes, mais reconnaissent à chaque fois que le dernier mot leur reviendra. Ce numéro d'équilibriste prend davantage de sens quand il est présenté par des personnages auxquels le public s'attache.

Cela a pu passer inaperçu aux yeux du lecteur occidental davantage concentré sur le contexte narratif (la compétition pour devenir numéro un, les histoires d'amour), mais le manga met en scène ce modèle de circulation de la connaissance en entreprise, établi par Nonaka et Takeuchi.

En présentant les différentes étapes dans la création de mangas, Bakuman montre au lecteur ce qui ne peut être formulé explicitement. On ne peut affirmer d'emblée que le scénariste ait voulu illustrer la théorie du management de Nonaka et Takeuchi; on constate toutefois qu'il met en scène les méthodes de management à la japonaise.

\section{f) Préparer dès aujourd'hui la nouvelle génération}

Bakuman fut un succès commercial, classé au top dix des ventes au Japon. Cela suffit déjà à justifier son existence, mais nous posons l'hypothèse que le Weekly Shonen Jump a édité Bakuman avec un objectif interne : préparer les nouveaux employés aux méthodes de travail, tout comme Canon et Matsushita employaient l'outil du journal interne.

Comment une entreprise peut-elle transmettre une vision d'ensemble? Comment peut-elle s'assurer de présenter à chaque employé, de chaque département, la vision et les savoir-faire des autres employés? Comment une tactique industrielle peut-elle mettre «l'art» au service de l'entreprise? Par le biais de la narration.

Le manga permet à tous les membres de la Shüeisha de comprendre le travail des collègues et prépare l'avenir : grâce au manga Bakuman, tous les futurs employés de la compagnie, les étudiants qui terminent leurs études et les écoliers qui débutent, disposent déjà des informations essentielles sur les techniques et l'organisation, d'où un gain de temps phénoménal. On peut imaginer que la nouvelle génération sera plus performante que les précédentes : elle connaîtra déjà les rôles et fonctions de chacun ainsi que les méthodes de travail.

L'approche japonaise, par ses concepts et ses objectifs, diffère de la vision occidentale et l'assume pleinement, même si, comme on l'a vu, une large composante demeure tacite, non formulée explicitement. Les entreprises produisant les mangas visent le meilleur système à tous les échelons : si l'artiste occupe une place essentielle, il est une pièce du système,

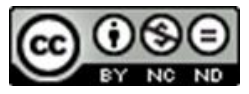


un organe de l'être vivant que forme la compagnie. Cette conception se comprend par l'étude du passé.

\section{Le déterminisme historique}

L'histoire du manga est inséparable de celle du Japon, ce qui pourrait expliquer pourquoi les autres pays qui ont tenté de se le réapproprier ont rencontré des difficultés ${ }^{24}$. Les exemples d'Ozamu Tezuka, mais aussi des pionniers du Gekiga à Osaka illustrent ce déterminisme. L'article s'appuie sur les ouvrages autobiographiques desdits auteurs : ces déclarations n'ont valeur que de «témoignage » et non de source objective toutefois elles traduisent la vision et le ressenti des acteurs du milieu.

Il serait tentant d'établir un parallèle entre l'évolution des mentalités et l'évolution des mangas (des contestations de 1968 à la société consommatrice hédoniste) : les générations post-guerre mettent en scène des héros adolescents, souvent orphelins, affrontant un monde hostile, sans l'aide des adultes « disparus », traduisant les traumatismes de la défaite et de l'holocauste nucléaire. La suprématie de la science transparaît dans les mangas des années cinquante, par exemple Tetsuwan Atomu (Astro Boy) ${ }^{25}$ en 1952 et Tetsujin 28-go (L'homme d'acier $\left.\mathrm{n}^{\circ} 28\right)^{26}$ en 1956 (base du genre mécha) : l'arme nucléaire a mis fin à la guerre malgré la détermination des Japonais.

Des auteurs comme Go Nagai communiquent une vision négative, voire tragique du monde et la défiance vis-à-vis des adultes et des autorités classiques. Les mangas des années 60 et 70 répercutent les revendications sociales. Enfin, les auteurs nés dans les années 80 , quand le Japon est redevenu un pays riche, une société hédoniste, s'éloignent des thèmes de la guerre et des problématiques sociales pour se recentrer sur le divertissement.

En sus de l'histoire des mentalités et des représentations, il serait pertinent de montrer comment les conditions «matérielles » ont déterminé le développement du manga. Le Japon accusait un important retard en imprimerie à cause de ses alphabets (trop compliqués). La modernisation sous l'ère Meiji (1868-1912) avec la lithographie puis l'offset aurait permis selon Bouissou (47) de faire de l'illustration un média de masse.

\footnotetext{
${ }^{24}$ Si le présent article s’intéresse à la France, d'autres études ont été menées sur la question du « global manga » et les tentatives infructueuses, sur les cinq continents, de localiser la production de mangas. Cf. Brienza, Casey.

25 Tetsuwan Atomu (Astro Boy) d’Ozamu Tezuka, pré-publié dans Shōnen de 1952 à 1968.

Deux traductions françaises disponibles : en 12 volumes chez Glénat et une anthologie de 6 volumes chez Kana.

26 Tetsujin 28-go (L'homme d'acier $\left.n^{\circ} 28\right)$ de Mitsuteru Yokoyama fut pré-publié dans Shônen de 1956 à 1966.
} 
Le manga est aussi devenu un divertissement pour enfants. Il paraît à côté de romans-feuilletons, d'articles éducatifs et de reportages, dans de gros magazines mensuels, comptant parfois plus de 200 pages, sous forme d'épisodes de quelques pages dont chacune constitue une histoire complète, mais dont les héros sont récurrents. Kôdansha, le plus grand éditeur japonais, crée la première gamme complète de magazines pour la jeunesse en lançant Shônen Club, destiné aux garçons jusqu'à l'entrée au collège (1914), puis Shôjo Club pour les filles (1923) et Yônen Club pour les plus jeunes (1926). Kôdansha est aussi le premier à rééditer les séries qui ont le plus de succès en magazine sous forme d'ouvrages brochés (tankôbon). (Bouissou 52-53)

Dès la première moitié du vingtième siècle apparaît la classification des productions en fonction du public cible comme le fit Hachette en France avec les collections verte (pour garçons) et rose (pour filles).

La bande dessinée japonaise n'est pas encore le manga tel que nous le connaissons. Néanmoins, elle présente déjà certaines spécificités qui la distinguent de notre BD et des comics américains, à commencer par son mode de production. Il s'agit d'une véritable industrie aux mains des grands éditeurs généralistes, tel Kôdansha, alors qu'en Occident ceux-ci ne s'y intéressent pas. Cette industrie a rapidement créé un marché de masse. En 1931, Yônen Club frôle le million d'exemplaires, et Kôdansha aurait réédité pas moins de cent fois en tankôbon « Le voyage de Dango Kushisuke » [Dango Kushisuke man'yuki], de Shigeo Miyao, entre 1924 et 1934. (Bouissou 53)

Ainsi on peut conclure que la construction du manga comme industrie rationnellement organisée débute dans la première moitié du vingtième siècle. Toutefois Tezuka, surnommé le « dieu du manga » (Brient 5-26) au Japon, va apporter ses codes au genre en le faisant évoluer : il écrit au début de la première version de Lost World (Le monde perdu) «Ce n'est ni un manga, ni un roman » (Brient 43). Hormis les apports artistiques, déjà étudiés (Hébert 139-151), il serait intéressant de voir comment les créateurs japonais ont, sans moyen, dans le Japon dévasté d'après-guerre, transformé un genre. Tezuka débute en 1946 dans un quotidien d'Ôsaka pour écoliers, puis enchaîne avec Hello Manga et va produire en akahon, des livres bon marché disponibles partout.

\section{L'expédient dans le dénuement}

Le manga en tant qu'objet est né d'une nécessité technique et économique, ainsi l'impression noir et blanc, de mauvaise qualité, sur papier jetable, pourrait apparaître comme un pis-aller, mais résulte d'une logique.

\section{a) Chercher le coût moindre}

La page de manga coûte 8 fois moins que l'européenne. « Il est 3,8 fois moins cher dans le cas le plus favorable à la BD (un album de 48 pages et un manga de 180 pages vendu chacun 7,50€), et 8,3 fois dans un des plus défavorables (un album de 52 pages vendu $13 €$ et un manga de 250 pages 
vendu 7,50 €). ( (Bouissou 410). Comment les Japonais sont-ils parvenus à ces tarifs compétitifs?

\section{b) Librairies de location « Kashihonya »}

Le Japon est ruiné par la guerre : acheter des albums (150 yens environ) serait un luxe. Se présente une solution de rechange : la location (10 yens par jour) :

Au début des années 1950, permettant de contourner le problème du coût d'achat trop élevé des mangas, même quand il s'agit d'akahon ou d'une revue mensuelle, un réseau de librairies de prêt, les kasbihonya, connaît un nouvel essor à partir de la région d'Ôsaka, et atteint même le nombre de 300000 [librairies] au milieu de la décennie.

D'ailleurs, les éditeurs d'akabon se mettent à produire pour les kachihonya afin de se trouver un autre marché que celui déclinant, du manga populaire à bas prix. Les librairies de prêt diffusent donc leurs propres œuvres (kashion manga) destinées non pas à être vendues, mais louées. Ainsi, toute une série d'auteurs vient de ce circuit de distribution. C'est en 1957, en réaction aux mangas pour enfants et au style issu des séries d’Ozamu Tezuka, qu'un nouveau genre de bande dessinée y fait son apparition. (Brient 17).

\section{c) «Choix » de l'encre noire}

Tezuka publie en noir et blanc pour l'efficacité à double titre, le coût moindre et la rapidité d'impression: " les planches en couleurs sont plus longues à imprimer que celles qui sont en bichromie qui elles-mêmes demandent plus de temps que celles simplement à l'encre de Chine » (Brient 11).

Problème de l'encre: «à l'époque, dans les livres destinés au prêt, les premières pages étaient imprimées en quadrichromie (16 pages) puis avec une encre différente toutes les 32 pages: violet, rose, vert, bleu, etc.» (Matsumoto 67). Alors que le choix du noir aurait pu résulter d'une volonté artistique (associer la couleur au polar), elle découle principalement de contraintes techniques.

Dans les années 50, la location coûte moins que la vente, mais dans la décennie suivante, les Japonais sont assez riches pour acheter et la demande ne cesse d'augmenter. La production suit: le système des ateliers et des assistants permet d'aller plus vite en décomposant les tâches. Cette division du travail n'est pas propre aux Japonais : Will Eisner rapporte dans son récit autobiographique The Dreamer (Le Rêveur) une organisation similaire de son studio aux É.-U. pour les comics. Côté franco-belge, l'isolement n'est pas non plus la norme: Hergé, l'auteur de Tintin engage des assistants dont Edgar-Pierre Jacobs, créateur de Blake et Mortimer.

En 1950, le dessinateur fonde les studios Hergé qu'animeront Bob De Moor [...], Jacques Martin, Roger Leloup et d'autres. Dès lors, il met au point une méthode rigoureuse : il conçoit de nouveaux récits, dont il étudie le découpage et établit, 
seul, les crayonnés. Il laisse ensuite à ses assistants le soin de préciser décors et accessoires [...] Il assure également l'essentiel de la mise à l'encre, leur laissant généralement le soin de parachever les détails. (Moliterni et al. 37)

Cette division du travail (dans une logique tayloriste) n'est pas l'apanage des ateliers japonais, toutefois ce trait mérite d'être souligné, car il ajoute à la dichotomie travail artisanal/industriel.

\section{d) L'obsession pour l'économie d'argent et le média-mix}

Bouissou (99) fait remonter la tradition japonaise du cross-média aux premiers produits dérivés datant de 1902 : les cartes à jouer et les poupées. On pourrait objecter qu'il s'agit de merchandising, à savoir la vente de gadgets superflus qui capitalisent sur la popularité d'une licence, toutefois il convient de rappeler que cross-média, média-mix et transmédia ambitionnent de développer des univers qui se déclinent sur plusieurs supports dans une logique créative.

La date clé de ce courant Média-mix japonais serait le $1^{\text {er }}$ janvier 1963, les premiers épisodes d'Astroboy à la télévision, qui marque le début d'une longue collaboration entre les différents médias. «Le facteur principal de cette synergie entre manga et télévision a été la diminution très importante du coût de production des séries animées. Tezuka y parvint en systématisant les techniques d'animation limitées. » (Bouissou 98). Le mangaka admire le travail de Disney, mais lui reproche de mal gérer l'argent : «J'ai un très grand respect pour Walt Disney, parce qu'il est une sorte de dieu du dessin animé. Pourtant, sa façon de réaliser des films d'animation présente de gros défauts. Le premier d'entre eux, c'est que ses dessins animés coûtaient énormément d'argent » (Tezuka 120).

Tezuka obtint ainsi de réaliser Astro avec 1200 planches au lieu de 17000 (soit quatorze fois moins) : « Pour créer les mouvements de Astro le petit robot, chaque planche sert pour trois images. Trois images identiques, puis trois images identiques, et ainsi de suite.» (Tezuka 122).

La collaboration se poursuit avec l'intégration d'autres secteurs au point que les fabricants de jouets peuvent intervenir dès la conception de nouvelles séries : «Bandai pria ainsi Nagai de doter ses robots de membres inférieurs massifs propres à assurer la stabilité des modèles réduits dont le grand fabricant se proposait d'inonder le marché.»(Bouissou 99). Goldorak est pensé à la fois comme manga, série animée et jouet.

Avec la crise de 1990 (causée par les séries formatées, le manque d'originalité des créateurs, la surproduction et surtout la diminution de la population) le manga continue de se réinventer avec des stratégies commerciales cross-média :

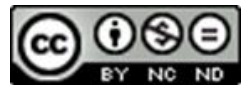


[...] dès 1993, Shûeisha a lancé V Jump, consacré aux jeux vidéo et aux séries manga qui en sont tirées (Dragon Quest) et Koroko systématise le doublonnage entre jeux et séries dessinées. Les grands médias-mix ludiques destinés aux jeunes, tels que Yu-Gi-Ob!, les Pokémon ou Card Captor Sakura, sont désormais à base de jeux vidéo, de cartes, de séries animées télévisées et de films : le manga n’en constitue plus qu'un élément secondaire. (Bouissou 144-145).

Les magazines demeurent (malgré la crise) :

[...] la médiocrité de l'impression et la publicité permettent aux magazines de garder un point d'équilibre assez bas (10 000 exemplaires pour un titre spécialisé, 30000 pour les ladies comics) ce qui limite les risques financiers. Ils ne sont donc pas prêts de disparaitre, comme l'atteste, même s'il s'est soldé par des échecs, le lancement des premiers gratuits en 2007. » (Bouissou 142).

En conclusion, les Japonais ont choisi depuis plusieurs décennies de privilégier l'efficacité en visant la production à bas coût et les succès commerciaux, que ce soit avec le choix de l'encre, le système des ateliers ou les stratégies média-mix. Il convient de souligner que cette dernière fut d'abord pensée comme adaptation d'un succès avant de devenir un choix initial : la création d'un univers décliné sur plusieurs matériaux.

Si la France a réussi des projets crossmédia comme le Dofus ${ }^{27}$ d'Ankama, elle reste sur l'idée d'adapter une licence populaire. Dorison estime qu'il faudrait un « succès à 300000 pour l'envisager » et que de nombreux artistes «y pensent déjà », mais que les éditeurs de $\mathrm{BD}$ « n’ont pas cette culture crossmédia» («Entretien du 8 février $2016 »)$. Hormis le projet Fusion d'Alain Damasio (actusf.com) — qui vise à créer d'emblée un roman, un film, un jeu vidéo, une série télévisée et un manga autour d'un même concept (la mémoire de l'eau) — peu d'artistes français envisagent le format transmédia dès la conception.

\section{Constat empirique des échecs et succès français}

En France, les échecs commerciaux n'établissent pas de droit, mais de fait une impossible transposition des modèles, notamment en raison des différentes conceptions, depuis la première tentative, l'Autoroute du soleil du français Baru dans Weekly Morning en 1994 — « l'expérience a achoppé sur la difficulté des participants à se plier au rythme de publication du manga, et sur leur connaissance trop limitée des attentes du lecteur japonais » (Bouissou 137) — jusqu'à Shogunmag.

Guillaume Dorison, directeur éditorial du projet, retient également la question du rythme (la législation française ne permet pas le système des ateliers et des assistants comme au Japon), l'absence de temps pour faire

27 Dofus est à l'origine un jeu vidéo français, un MMORPG sorti en 2004 dont le succès a permis la création d'autres jeux, d'une série de dessins animés, d'une série de mangas et même un film en 2015. 
éclore le talent de certains auteurs à fort potentiel, mais surtout les problèmes financiers des Humanoïdes Associés au moment des années 2008-2009 qui ont dû stopper l'expérience (« Entretien du 8 février 2016).

Par conséquent, il serait prématuré de tirer des conclusions de cette tentative, arrêtée pour des raisons contingentes. D'autant que certains manfras ont finalement trouvé leur public.

Pour savoir ce que valent vraiment les magazines de prépublication en France, il faudrait mener l'expérience sur plusieurs années avec différentes revues.

Les manfras peuvent s'arrêter pour différents motifs: une qualité graphique jugée insuffisante par le public, l'absence de marché ou encore le rythme.

Il convient toutefois de nuancer notre thèse en analysant quelques cas de manfra à succès et les causes de leurs réussites, laissant entrevoir un futur manga mondial.

\section{1) La copie habile des codes japonais}

Certains artistes français, comme Jenny, ont réussi à produire un manga français en reproduisant les codes, graphiques et narratifs, et en situant l'histoire au Japon : Pink diary (8 volumes).

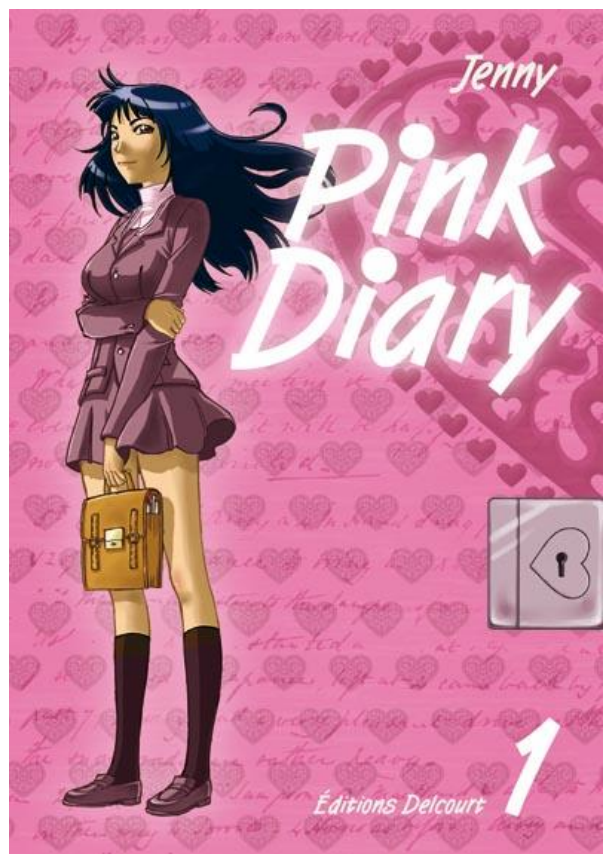

Pink Diary, volume 1, Jenny

(C) Éditions Delcourt, 2006

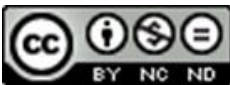




\section{2) Stratégie mix-media}

La compagnie française Ankama présente un exemple de mix-média réussi : à partir du succès du jeu vidéo Dofus (dont la charte graphique s'inspire déjà du style manga), Vincent Deruyck, « Ancestral-Z », conçoit le manga (22 volumes minimum). L'auteur explique la réception positive de ce manga par «la série Dofus ou le Jeu et la licence [qui] poussent les gens à essayer de le lire» (« Entretien du 27 janvier $2016 »)$.

De rares projets français comme le Fusion de Damasio envisagent la création d'un manga en parallèle à l'univers et non en amont.

\section{3) Hybridation: La BD européenne qui s'inspire du manga, mais fait de la BD}

Les légendaires (23 albums) de Patrick Sobral et La Rose écarlate (11 albums) de Patricia Lyfoung présentent des exemples réussis d'hybridation: des bandes dessinées au format franco-belge, avec des inspirations japonisantes.

\section{4) Artiste radicant: au-delà de l'bybridation}

Nicolas Bourriaud, ancien directeur de l'École nationale supérieure des Beaux-arts de Paris, défend la théorie d'une nouvelle étape « globalisée » dans l'histoire de l'art, après le post-modernisme qu'il date de l'exposition «Les Magiciens de la Terre $»^{28}$ et qui s'appuyait sur l'idée que «l'origine prime [...] sur la destination dans la vie des formes et des idées » (31).

Ce que le post-moderne nomme bybridation consiste à greffer sur le tronc d'une culture populaire devenue uniforme des "spécificités» la plupart du temps caricaturales, comme on parfume avec différents flavours synthétiques les sucreries industrielles. Seuls deux modèles semblent aujourd'hui s'opposer à ces facilités, eux-mêmes contradictoires : d'un côté le repli identitaire, crispation sur des valeurs esthétiques traditionnelles et locales, et de l'autre ce que l'on appelle la créolisation, sur le modèle caribéen d'acclimatation et de croisements d'influences hétérogènes. (Bourriaud, 21).

Contre cette hybridation qui se réduirait à une combinaison de signes, dénués de sens, se dessine la solution isolationniste, l'enfermement dans les « racines ». Toutefois, les artistes peuvent refuser ce déterminisme pour choisir de se réapproprier les influences extérieures et assimiler les racines d'autres cultures « car les créateurs contemporains posent déjà les bases d'un art radicant - épithète désignant un organisme qui fait pousser ses racines et se les ajoute, au fur et à mesure qu’il avance. » (23).

2818 mai 1989, conçue par Jean-Hubert Martin, au centre Pompidou et à la Grande Halle de la Villette, Paris, 1989. 
L'effet pervers de l'échec du multiculturalisme postmoderne à inventer une alternative à l'universalisme moderniste, pour Bourriaud, serait qu'un travail d'artiste serait inéluctablement expliqué par la condition, le statut ou l'origine de son auteur: par exemple, le franco-belge fait de la $\mathrm{BD}$, l'américain du comics et le japonais du manga parce que ce sont leurs racines et que le marché de l'art ne souhaite pas savoir ce qu'ils veulent faire : «Pourquoi un artiste iranien, chinois ou patagon se verrait-il sommé de produire sa différence culturelle dans ses œuvres, tandis qu'un Américain ou un Allemand serait davantage jugé sur sa critique des modèles de pensée et sa résistance aux injonctions du pouvoir et aux diktats des conventions? » (31).

L'œuvre est interprétée en fonction de son origine et non pas de son intention, avec le risque d'un réductionnisme en faisant des créations de simples produits de leur époque, prédictibles, et non des acteurs agissants : Une œuvre de Kazimir Malevitch ou de Marcel Duchamp ne peut pas être considérée uniquement comme un produit de l'Histoire et des circonstances sociopolitiques qui l'ont vu naitre : elle constitue également un événement qui génère des effets, influence son époque, bref produit l'histoire, autant qu'elle est le résultat logique d'une série de déterminismes. (40-41)

En ce sens, des auteurs sémionautes, «d'origine française », offrent des exemples de titres de mangas radicants réussis : City hall ${ }^{9}$ ( 7 volumes), dont l'univers steampunk recycle les références de la littérature française et Dreamland $^{30}$ (14 volumes), qui se déroulent tous deux en Europe. Le manga Radiant du français Tony Valente est exporté et diffusé au Japon par Asukashinsha.

\section{5) Vers un manfra digital?}

Enfin une piste envisagée au Japon, le digital manga, lisible en ligne, se développe également en France dans un style propre. Les plateformes non professionnelles comme Amilova accueillent des tentatives comme Nail Saga de Dave-X ou Chronoctis Express d'Aerinn. Ce modèle reproduit les avantages de la prépublication : délais raccourcis, visibilité pour les auteurs, retours des lecteurs (commentaires) et ajustements possibles.

\footnotetext{
${ }^{29}$ City Hall de Rémy Perrin et Guillaume Lapeyre est publié par Ankama depuis 2012 et compte 7 volumes.

30 Dreamland de Reno Lemaire est publié par Pika Edition depuis 2006 et compte déjà 14 volumes.
} 


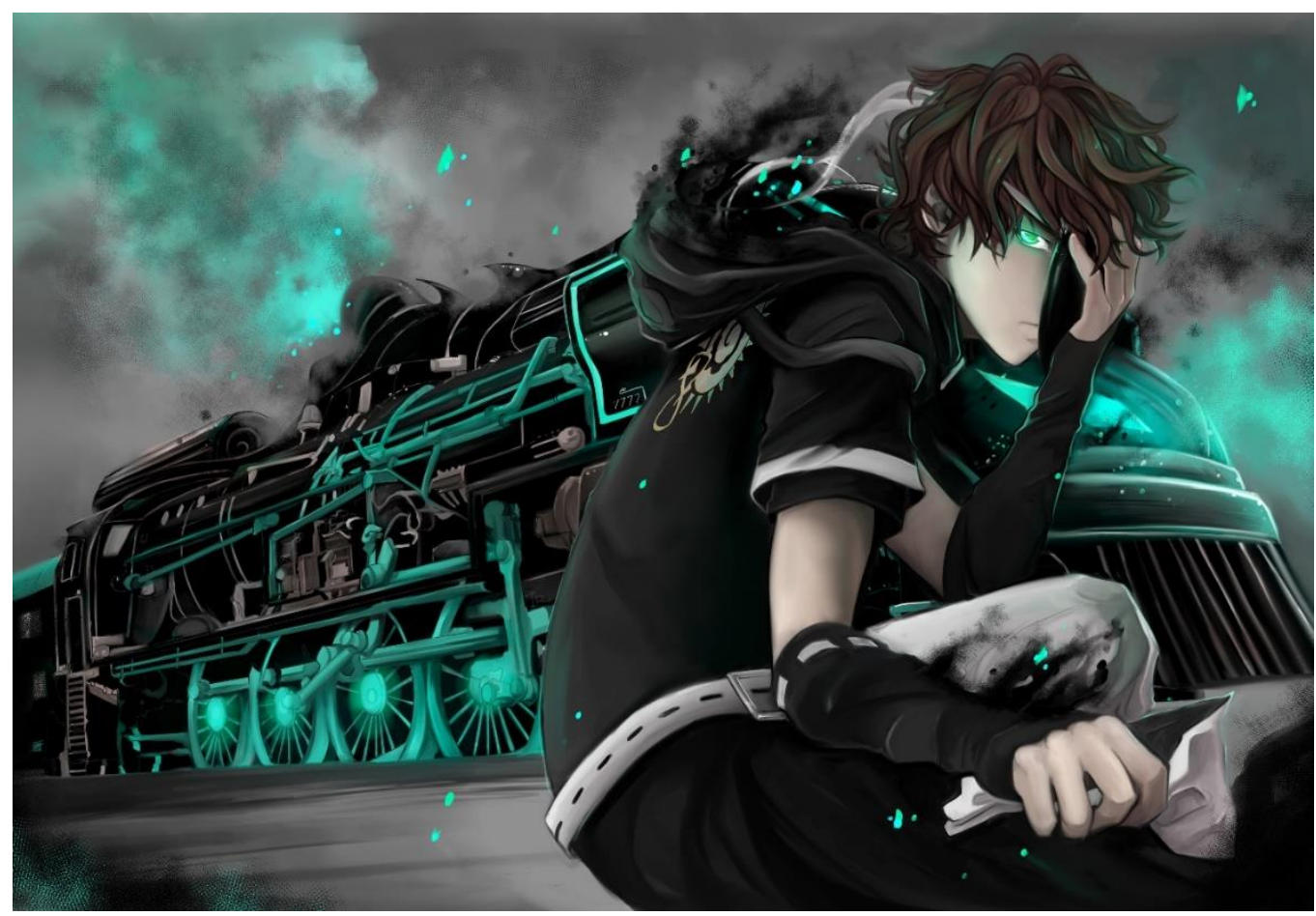

Chronoctis Express, jaquette - Aerinn.

(C) 2016.

\section{Conclusion}

En conclusion, malgré une réception favorable du manga japonais en France, la création originale a rencontré de nombreux obstacles. Nous défendons la thèse que le manga est indissociable de son contexte d'origine. D'abord, nous avons constaté que le manga est présenté par ses créateurs et les compagnies comme une entreprise commerciale assumant sa recherche d'efficacité. Puis, nous avons établi que la création du manga (format et thème) est fortement déterminée historiquement, c'est pourquoi la reproduction par d'autres pays a présenté des difficultés. Ensuite, nous avons vu que la création « à la japonaise » est une série d'expédients, de trouvailles inventées pour des raisons plus commerciales qu'artistiques. Enfin les échecs français attestent de fait et non en droit cette impossible transposition.

Nous l'expliquons notamment par deux visions radicalement opposées : alors que beaucoup d'Européens cherchent l'art pour l'art, les Japonais assument leur recherche d'efficacité commerciale et l'organisation industrielle.

Néanmoins la thèse post-moderne visant à expliquer une création par son seul contexte et ses racines doit être nuancée notamment en observant les succès français: par la copie des codes graphiques et narratifs, la

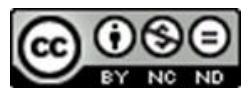


reproduction de la stratégie commerciale, l'intégration des influences ou encore la capacité à se réapproprier les racines étrangères.

Si les artistes français ont su copier ou se réapproprier les codes graphiques et narratifs, la reproduction du système japonais de création n'a pas encore été effectuée et il est impossible de conclure, en l'absence d'observation, si oui ou non, les Français pourraient réussir au même titre que les Japonais avec un contexte équivalent. 


\section{Bibliographie ${ }^{31}$}

« Aerinn », Entretien du 1er février 2016.

Arendt, Hannah. La crise de la culture.1961-1968. Paris : Gallimard, coll. «Folio », 1972.

Association des éditeurs coréens Korean Publishers Association (KPA) ; KPA. www.kpa21.or.kr. Consulté le $1^{\text {er }}$ février 2016.

Barulea, Hervé (Baru). L’Autoroute du soleil (Taiyô Kôsoku). Morning, 1994.

Bouissou, Jean-Marc. Histoire et univers de la bande dessinée japonaise. 2010. Paris : Philippe Picquier, réédition poche 2014.

Bouissou, Jean-Marc, Dolle-Weinkauff, Bernd et Pelliteri, Marco. «Manga in Europe. A short study of market and fandom ", JohnsonWood, Toni. (dir.) Manga. An Anthology of Global and Cultural Prospectives, Continuum International, 2010.

Bourriaud, Nicolas. Radicant. Pour une esthétique de la globalisation. Paris: Denoël, 2009.

Brient, Hervé. «Osamu Tezuka : dissection d'un mythe », Manga 10000 images, no2. Versailles : Ed. H, 2009.

Brienza, Casey. Global Manga. Japanese comics without Japan, City University London, UK, 2015.

Deruyck Vincent (Ancestral Z) ; sc. Roux, Anthony (Tot). Dofus. Ankama Editions, 2005-2016.

Deruyck Vincent (Ancestral Z), Entretien du 27 janvier 2016.

Dorison, Guillaume (Izu). Entretien du 8 février 2016.

Eisner, Will. The Dreamer (Le Rêveur), Kitchen Sink Press, 1986.

Hébert, Xavier. "Tezuka Osamu (1928-1989), esprit novateur du manga », Ebisu, Volume 27, no.1, 2001, pp. 139-151.

Gautier, Théophile., Mademoiselle de Maupin. 1834. Paris : Folio, Collection Folio classique, 1973.

Inada, Kôji ; sc. Sanjô, Riku. Dragon Quest. Dai no bôken (Dragon Quest ou La quête de Daï. Shônen Jump, 1989-1996.

Ishinomori, Shôtarô. Nihon manga keizai nyûmon (Les Secrets de l'économie japonaise en bande dessinée). Nibon Keirai Sbinbun, 1986-1989.

Japan External Trade Organization, Content Industry in Japan. 2007. Tokyo, JETRO, 2008.

Jenny. Pink Diary. Delcourt, 2006-2008.

Kant, Emmanuel. Critique de la faculté de juger. 1790. CEuvres philosophiques, tome 2, Paris : Gallimard, Bibliothèque de la Pléiade, no.317, 1985.

Khanna Tarun. «L'intelligence contextuelle », Harvard Business Review France no.11, oct-nov 2015, pp. 70-80.

Kôjima, Gôseki ; sc. Koike, Kazuo. Kozure ôkami (Lone Wolf and Cub). Manga Action, 1970-1976.

\footnotetext{
${ }^{31}$ Le titre français des mangas est indiqué entre parenthèses pour les séries traduites, sauf
} si le titre est en anglais ou commun aux deux langues. 
Kurumada, Masami. Saint Seiya (Les Chevaliers du zodiaque). Shônen Jump, 1986-1990.

Lardellier Pascal. «Ce que nous disent les mangas », Le Monde Diplomatique, décembre 1996, p. 29.

Lemaire, Reno. Dreamland. Pika Edition, 2006-2016.

Lyfoung, Patricia. La Rose écarlate, Delcourt, 2005-2016.

Matsumoto, Masahiko. Gekiga Fanatics. 1979. Traduction française de Slocombe Miyako. Poitiers : Le lézard noir, 2013.

Mauguière, Claire. L'édition en Corée du Sud, Département études du Bureau Internationale de l'Edition Française, avril 2010.

Matsumoto, Leiji (Akira). Uchû kairoku Captain Hârokku (Capitaine Albator/Captain Harlock), Play Comic, 1977-1979.

Mayo, Anthony, Nohria, Nitin et Rennella, Mark. Entrepreneurs, Managers, and Leaders: What the Airline Industry Can Teach Us About Leadership. New York: Palgrave Macmillan, 2009.

Mayo, Anthony, Nohria, Nitin et Singleton, G. Laura. Paths to Power: How Insiders and Outsiders Shaped American Business Leadership. Boston: Harvard Business School Press, 2007.

Mayo, Anthony et Nohria, Nitin. In Their Time: The Greatest Business Leaders of the 20th Century. Boston, MA: Harvard Business School Press, 2005.

Miyao, Shigeo. Dango Kushisuke man'yûki (Le voyage de Dango Kushisuke). Tôkyô Maiyû Shinbun, 1924-1934.

Moliterni, Claude, Mellot, Philippe et Denni Michel. Les aventures de la BD, Paris : Gallimard, Découverte Littérature, 1996.

Nagai, Gô (Kiyoshi). UFO Robot Grendizer (Goldorak). Telebi Magazine, 19751976.

Nonaka Ikujiro et Takeuchi Hirotaka. The Knowledge-Creating Company, New York: Oxford University Press, 1995.

Obata, Takeshi ; sc. Ohba, Tsugumi. Death Note. Shônen Jump, 2004-2006.

Obata, Takeshi ; sc. Ohba, Tsugumi . Bakuman. Weekly Shônen Jump, 20062008.

Perrin, Rémy et Lapeyre, Guillaume. City hall. Ankama, 2012-2015.

Polanyi, Michael. The tacit dimension, Gloucester : Mass, Peter Smith, 1983.

Ratier, Gilles. Une année de bande dessinée, rapport annuel de l'Association des Critiques de Bande Dessinée. 2015.

Rieu Alain-Marc. «Le Japon comme société de connaissance : quelles leçons pour la France? ", Sabouret, Jean-François (sous la direction de), L'empire de l'intelligence: les politiques de recherche japonaises depuis 1945, Paris : CNRS Editions, 2007, pp. 185-216.

Royal, Ségolène. Le ras-le-bol des bébés zappeurs. Télémassacre: l'overdose, Paris : Robert Laffont, 1989.

Sobral, Patrick. Les Légendaires, Delcourt, 2004-2016.

Takahashi, Kazuki. Yu-Gi-Ob!, Shônen Jump, 1996-2004.

Takahasi, Yôichi. Captain Tsubasa. Shônen Jump, 1981-1988.

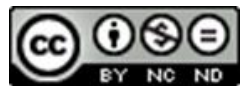


Tanaka, Akio ; sc. Hashimoto, Izô. Shamo (Coq de combat). Manga Action, puis Evening depuis 1998.

Taniguchi Jirô ; sc. Sekiwa, Natsuo. Haruka na machi e (Quartier Lointain). Manga Action, 1986-1998.

Taniguchi, Jirô ; sc. Yamemakura, Baku. Kamigami no itadaki (Le Sommet des dieux), Business Jump, 2000-2003.

Tezuka, Osamu ; sc. Sakai Schichima. Shin Takarajima (La Nouvelle Île au Trésor). Ikkuei Shuppan, 1947.

Tezuka, Osamu. Zenseiki (Lost World). Fuji shobô, 1948.

Tezuka, Osamu. Tetsuwan Atomu (Astro boy). Shônen. 1952-1968.

Tezuka, Osamu. Ma vie Manga, Bruxelles : Kana, coll. «Sensei », DL 2011.

Toriyama, Akira. Dragon Ball. Shônen Jump, 1984-1995.

Toriyama, Akira. Hetappi : Crummy Manga Lab (L'apprenti mangaka). Flesh Jump, 1984.

Trécourt, Fabien. «La métaphysique des mangas », Sciences Humaines, no.256, Février 2014.

Valente, Tony. Radiant. Ankama 2013-2016.

Vincent, Jérôme, actusf.com [en ligne], 2015,

http://www.actusf.com/spip/Interview-2015-Alain-Damasio-pour.html Consulté le 12 février 2016.

Yokoyama, Mitsuteru, Tetsujin 28-go (L'homme d'acier n²8). Shônen, 19561966. 